\title{
Dysregulation of autophagy and mitochondrial function in Parkinson's disease
}

\author{
Bao Wang ${ }^{1,2}$, Neeta Abraham², Guodong Gao ${ }^{1}$ and Qian Yang ${ }^{1 *}$
}

\begin{abstract}
Parkinson's disease (PD) is the second most common neurodegenerative disease. Increasing evidence supports that dysregulation of autophagy and mitochondrial function are closely related with PD pathogenesis. In this review, we briefly summarized autophagy pathway, which consists of macroautophagy, microautophagy and chaperone-mediated autophagy (CMA). Then, we discussed the involvement of mitochondrial dysfunction in PD pathogenesis. We specifically reviewed the recent developments in the relationship among several PD related genes, autophagy and mitochondrial dysfunction, followed by the therapeutic implications of these pathways. In conclusion, we propose that autophagy activity and mitochondrial homeostasis are of high importance in the pathogenesis of PD. Better understanding of these pathways can shed light on the novel therapeutic methods for PD prevention and amelioration.
\end{abstract}

Keywords: Parkinson's disease, Autophagy, Macroautophagy, Mitophagy, Chaperone-mediated autophagy, Mitochondria,a-synuclein, PINK/Parkin, LRRK2, DJ-1

\section{Background}

Parkinson's disease (PD) is the second most common neurodegenerative disease. Although most of PD cases are sporadic, more than $5 \%$ are inherited [1]. The PD related genes mainly include (1) $S N C A$, the gene encoding $\alpha$-synuclein, which is the main component of Lewy bodies [2]; (2) LRRK2, which encodes a multi-domain large protein. Variable LRRK2 mutants contribute to over $10 \%$ of familial and about $3 \%$ of sporadic PD cases [3]; (3) PINK1 and Parkin, which are genes involved in mitochondrial turnover and maintenance. Their mutations are associated with early-onset PD [4]. (4) DJ-1, whose mutants contribute to $1-2 \%$ of autosomal recessive PD [5].

Given the role of autophagy in the elimination of cellular dysfunctional proteins/organelles, the contribution of impaired autphagy to PD has attracted increasing interest. Accumulating evidence indicates autophagy defects are involved in the PD pathogenesis. PD related VPS35

\footnotetext{
* Correspondence: qianyang@fmmu.edu.cn

${ }^{1}$ Department of Neurosurgery, Tangdu Hospital, The Fourth Military Medical University, No. 569 Xinsi Road, Baqiao District, Xi'an 710038, Shaanxi Province, China

Full list of author information is available at the end of the article
}

D620N mutant inhibits autophagy and impairs the trafficking of the autophagy protein ATG9A [6]. In addition, in the substantia nigra (SN) of PD patients, aberrant autophagy activity is identified [7]. Inducing autophagy by manipulating Polo-like kinase 2 or activiting chaperonemediated autophagy (CMA) can reduce $\alpha$-synuclein aggregation $[8,9]$. Finally, PD related neurotoxin 1methyl-4-phenyl-1,2,3,6-tetrahydropyridine (MPTP) can induce autophagy through CDK5-mediated phosphorylation of endophilin B1 [10]. Rotenone could impair mitophagy to damage neurons which can be rescued by DJ-1 overexpression [11].

Recently, increasing reports support the role of mitochondrial dysfunction in the degeneration of DA neurons during PD initiation and progression. Mitochondria are double membrane organelles and participate in multiple cellular processes, including calcium homeostasis, energy supply, metabolic synthesis and apoptosis [12]. Mutations in mtDNA are more frequent in PD patients when compared with the age-matched population [13]. Interestingly, one recent study reports that $\alpha$-synuclein overexpression augments mitochondrial $\mathrm{Ca}^{2+}$ transient by enhancing endoplasmic reticulum-mitochondria interactions and 
physiological levels of $\alpha$-synuclein are essential to maintain mitochondrial function and morphology [14]. MPTP and rotenone can disturb mitochondrial function, impair respiratory chain complex and induce oxidative stress $[15,16]$. Thus, excessive mitochondrial stress in response to environmental toxins or impaired capability of neurons to remove damaged mitochondria through mitophagy may result in the death of neurons.

This review discussed the recent findings in the relationship among several PD related genes, autophagy and mitochondrial dysfunction (Table 1).

\section{Autophagy}

Autophagy, which derived from the Greek words for "self-digesting", refers to the cellular catabolic process to remove the cytosolic components, including mainly dysfunctional organelles, misfolded proteins, and surplus or unnecessary cytoplasmic contents to lysosome for digestion [17].

Autophagy can be divided into three major types: macroautophagy, microautophagy and chaperone-mediated autophagy (CMA). This classification is dictated by the way in which the cellular components are being delivered to lysosomes [17].

Macroautophagy (MA) is the most common and well conserved form of autophagy, and enables the bulk degradation of cellular contents [18]. It mainly involves five steps: initiation, nucleation, elongation, fusion, and degradation [19]. In this process, the pre-autophagosomal structure (PAS) elongates from membrane of Golgi, mitochondria or endoplasmic reticulum and sequesters the cullular contents or organelles to form the autophagosome. Subsequently, the autophagosome fuses with lysosomes to allow the cargo to be degraded [20]. Mitophagy describes the clearance of damaged mitochondria by macroautophagy. Increasing evidence prove that dysregulation of mitophagy play important roles in neurodegenerative diseases [21].

Table 1 PD-related genes are involved in dysregulation of autophagy and mitochondria

\begin{tabular}{ll}
\hline a-synuclein & Macroautophagy \\
& Mitophagy \\
& CMA \\
PINK1/Parkin & Mitophagy \\
LRRK2 & Macroautophagy \\
& CMA \\
& Mitochondria \\
DJ-1 & Macroautophagy \\
& CMA \\
& Mitochondria \\
\hline
\end{tabular}

Microautophagy is the process in which cytoplastic contents are directly engulfed into lysosomes for degradation without an intermediate autophagosome [22]. Also, microautophagy is constitutionally active under normal conditions and maintains the turnover of cellular nutrients via selective degradation of proteins and organelles [23]. Unlike macroautophagy, nutritional deprivation or stress cannot activate microautphagy [24]. Currently, knowledge about the role and function of microautophagy, especially in neurodegenerative disease, is limited and further studies are needed.

CMA is characterized by the high selectivity in substrates targeting [25]. The substrates for CMA present a unique pentapeptide motif, which is known as KFERQ. Notably, this motif is a pattern recognition motif associated with the hydrophobicity and charge of amino acid residues, rather than $100 \%$ compliance with specific amino acid residues [26]. Interestingly, post-translational modification could perfect some KFERQ-similar motif for CMA through changing the hydrophobicity and charge of amino acid residues [27]. Briefly, CMA can be divided into four main steps: recognition, unfolding, translocation and degradation [28]. Cytoplastic heat shock cognate protein 70 (Hsc70) binds to the substrates after recognizing the KFERQ motif and delivers it to lysosomal surface, where the substrates could be unfolded with the help of Hsc70 and other chaperons. Afer that, the unfolded substrate is translocated into lysosome via lysosome associated membrane protein type 2A (LAMP2A) for degradation under the lysosomal acid environment.

\section{Mitochondrial dysfunction in Parkinson's disease}

Although the mechanism underlying PD pathogenesis is still unclear, mitochondrial dysfunction has been increasingly confirmed to be a vital contributor. The main characterizations of mitochondrial dysfunction include ROS overproduction, ATP depletion, mitochondrial DNA depletion, caspase release and electron transport complex (ETC.) enzyme defection [29]. Neurotoxins applied to establish PD models, including MPTP and rotenone, damage mitochondrial function by inhibiting complex I activity [30]. One recent study specifically knockouts $N d u f s 4$ gene in mice midbrain DA neurons and reports significant compromise in complex I activity. However, Ndufs4 deficit mice show no obvious neurodegeneration, no loss of striatal innervation and no movement impairment, but with increased vulnerability to MPTP, suggesting that impaired complex I activity is involved in the pathogenesis of PD [31]. Another study shows that complex I inhibition can lead to abnormal oxidition of optic atrophy 1 (OPA1), which in turn results in mitochondrial structural abnormalities and dopaminergic neurodegeneration. All these pathogenic changes are abolished by OPA1 upregulation, hinting that OPA1 is a novel therapeutic target for PD [32]. 
Mitophagy means the way in which damaged mitochondria are eliminated by autophagy. Through mitophagy, cells regulate both the number and quality of mitochondria in response to the metabolic stress. One recent study using genome-wide RNAi screen identifies sterol regulatory element binding transcription factor 1 (SREBF1), F-box and WD40 domain protein 7 (FBXW7) as key regulators for PINK/Parkin pathway, which is reponsible for mitophagy activition [33]. Moreover, SREBF1 is considered as a risk locus for sporadic PD [34]. Thus, this study hints that mitophagy may act as a common mechanistic link between sporadic and autosomal recessive PD. Mutation in F-box domaincontaining protein Fbxo7 is related with early-onset autosomal recessive PD. One study reports that Fbox7 is involved in mitochondrial maintainance via directly interaction with Parkin to induce mitophagy, which further confirms the importance of mitophagy in PD pathogenesis [35].

Our study shows that the myocyte enhancer factor 2D (MEF2D) is present in neuronal mitochondria and heat shock protein 70 (Hsp70) interacts with MEF2D and mediates its mitochondrial translocation. Mitochondrial MEF2D is responsible for the expression of the gene NADH dehydrogenase 6 (ND6), which encodes an essential component of the complex I. Blocking MEF2D function leads to impaired complex I activity, oxidative stress and neuronal death. More importantly, in the postmortem PD brain samples, both mitochondrial MEF2D and ND6 levels are decreased compared with age-matched controls [36]. Our further study shows that MPTP caused a significant decrease in the half-life and total level of MEF2D mRNA. Down-regulation of MEF2D mRNA alone can reduces the viability of SN4741 cells and sensitizes the cells to neurotoxin [37].

\section{PD-related genes are involved in dysregulation of autophagy and mitochondria a-synuclein}

$\alpha$-synuclein is encoded by $S N C A$ and its fibrillar form is the major component of Lowy bodies [38]. Existence of hydrophobic non-amyloid $\beta$ component domain endows $\alpha$-synuclein with the propensity to aggregate [39]. Aggregated $\alpha$-synuclein can trigger neuronal death in PD [40]. Thus, intensive researches focus on how to prevent or eliminate the aggregation of $\alpha$-synuclein. Study show that ubiquitin proteasome system (UPS) is responsible for degrading monoubiquitinated $\alpha$-synuclein, while macroautophagy is for removing deubiquitinated $\alpha$ synuclein [41, 42]. Further study demonstrates, in normal condition, $\alpha$-synuclein is mainly degraded by UPS [43]. Macroautophagy pathway can be activated in response to increased level of wild type or A53T mutant $\alpha$-synuclein [44, 45]. However, some studies show that increased $\alpha$-synuclein can impair autophagosome synthesis by inhibiting Rab1a [46]. Also, pre-formed $\alpha$-synuclein aggregates compromise autophagosome clearance and are resistant to degradation by autophagy [47]. The difference may be derived from the variability of models or different levels of $\alpha$-synuclein. Further studies are warranted to address such discrepancy.

Abnormal $\alpha$-synuclein level can also disrupt mitophagy. In PD postmortem brain tissues, $\alpha$-synuclein accumulation increases oxidative stress and disturbs mitochondrial function [48]. Moreover, both in vivo and in vitro, expression of $\alpha$-isoforms of $\alpha$-synuclein in neurons causes the fragmentation of mitochondria, which will eventually leads to the decline in respiration and neuronal death [49]. Over-expression of $\alpha$-synuclein can occur in mitochondria and disrupt mitochondrial membrane potential by opening the mitochondrial permeability transition pore (mPTP), thereby initiating mitophagy [45]. $\alpha$-synuclein can activate MPTP via interacting with either adenylate translocator (ANT) or voltage dependent anion channel (VDAC) [50, 51]. Further study found that A53T mutant could impair mitochondrial function by residing in mitochondria membrane as monomers and oligomers [52].

Increasing evidence confirms $\alpha$-synuclein as a target of chaperone mediated autophagy (CMA). Hsc70 can recognize soluble $\alpha$-synuclein and the affinity between Hsc70 and $\alpha$-synuclein fibrils is 5 -fold tighter compared with soluble $\alpha$-synuclein [53]. One recent study showed that Hsc70 and Ssa1p work like a tweezer to bind two domains within $\alpha$-synuclein [54]. Also, in vitro, one study shows the uptake of extracellular $\alpha$-synuclein by neurons and their retrograde axonal transportation to neuronal soma. However, Hsc70 chaperones $\alpha$-synuclein in the extracellular space and alleviates $\alpha$-synuclein oligomer formation [55]. Activating CMA activity via up-regulating LAMP2A decreases $\alpha$-synuclein turnover and protects against $\alpha$-synuclein over-expression induced neurotoxicity [9]. Our study showed MEF2D, a transcription activator identified as neuronal survival factor, is the substrate of CMA. Wild type or mutant $\alpha$-synuclein accumulation compromises normal turnover of MEF2D by CMA and leads to decrease in the MEF2D DNA binding ability and neuronal stress, which underlies the neuronal loss of PD [56]. Thus, enhancing CMA pathway could be a promising therapeutic strategy for PD treatment.

\section{PINK1/Parkin}

PINK1 is a serine/threonine kinase protein and mutations in PINK1 cause a rare form of autosomal recessive PD [57]. Parkin containing ubiquitin E3 ligase can ubiquitinate multiple substrates for degradation. Mutations of Parkin lead to accumulation of its substrates and are related with early onset juvenile autosomal recessive PD [58]. 
Increasing evidence suggests the PINK1/Parkin pathway is essential for mitochondrial quality control. One recent study shows that Parkin ubiquitinates dynaminrelated protein 1 (Drp1) to promote its degradation. Disruption of this interaction by Parkin mutation leads to the accumulation of Drp1 and mitochondrial fragmentation [59]. In Drosophila, both Parkin and PINK null mutants show a significant overall slowing of motichondrial protein turnover and mitophagy. Failure to remove the damaged mitochondrial proteins plays an important role in PD pathogenesis [60]. PINK1 mutation affects mitochondrial complex I activity and the maintainance of the electron transport chain, which disturbs the mitochondrial membrane potential [61]. Upregulation of Parkin protects cells against multiple stresses, including endoplasmic reticulum stress, mitochondrial stress, proteotoxicity and excitotoxicity [62, 63]. By contrast, loss of Parkin results in mitochondrial fragmentation, decreased cellular $\mathrm{Ca}^{2+}$ handling capability and increased cellular vulnerability to stress [64]. All the above findings demonstrate that deficiency in PINK1/Parkin pathway leads to mitochondrial dysregulation.

Recent researches on PINK1/Parkin pathway have revealed molecular details for mitochondria protection. Once the mitochondria are impaired and the membrane potential gets depolarized, PINK1 will accumulate on the outer membrane of mitochondria (OMM). A study showed dimeric PINK1 on OMM can recruit Parkin and thereby phosphorylates Parkin at Ser65 [65]. Research using mass spectrometry identified VDACs as the docking site for Parkin recruitment to the OMM [66]. After Parkin translocation to mitochondria, many OMM proteins are ubiquitinated by Parkin and in turn recruited other proteins to initiate mitophagy. Then, these Parkin labeled mitochondria are brought to lysosomes for degradation. This PINK1/Parkin signaling pathway can be positively modulated by AF-6, which is lacked in caudate/ putamen and SN of sporadic PD patients [67]. Moreover, up-regulation of translocation of the OMM (TOMM) can rescue mitophagy impaired by Parkin mutations, hinting that TOMM acts as an important regulator in PINK1/ Parkin mediated mitophagy [68]. Despite extensive research, how autophagy related proteins are recruited during mitophagy process is still unclear and to what extent mitophagy dysregulation contributes to PD pathogenesis remains to be investigated.

\section{LRRK2}

Leucine-rich repeat kinase 2 (LRRK2) is one of the generic contributors to PD. As estimated, variable LRRK2 mutants contribute to over $10 \%$ of familial and about $3 \%$ of sporadic PD cases [69]. So far, over 50 LRRK2 mutations have been identified in PD patients. Among these, the G2019S mutation is the most common cause of autosomal dominant familial PD cases [70]. Also, G2019S mutation can be found in about $2 \%$ sporadic PD. Thus, exploring LRRK2 pathogenicity is essential to understand the molecular mechanisms of PD.

Many reports show a relationship between LRRK2 and macroautophagy. In kidney of mouse, loss of LRRK2 leads to age-dependent bi-phasic alteration of macroautophagy activity [71]. In human neuroglioma cells, inhibition of LRRK2 kinase activity can stimulate macroautophagy in the absence of any alteration in mTOR pathway, suggesting that LRRK2 regulates autophagic activity independent of mTOR signalings [72]. Consistently, LRRK2 activates a persistent increase in autophagosome formation through a calcium associated pathway. Simultaneously, LRKR2 upregulation increases p62 and decreases the number of acidic lysosomes [73]. Moreover, G2019S mutation increases autophagic vacuoles and shortens neurite length [74]. The effects of G2019S on neurite length can be abolished by down-regulation of LC3 or Atg7 and enhanced by autophagy inducer rapamycin, hinting that autophagy plays an important role in regulation of neurite length [75]. In fibroblasts, G2019S LRRK2 mutant exacerbates MPTP-induced cell death dependent of autophagic activity [76]. Furthermore, a study showed that LRRK2 increases the number of autophagosomes by activating CaMKK/ AMPK pathway [73]. In addition, G2019S mutation augments autophagic flux by MEK/ERK signaling [77].

LRRK2 and the PD-associated mutations can be degraded by CMA. In normal condition, both UPS and CMA are responsible for the degradation of wild-type LRRK2. However, G2019S LRRK2 disrupts degradation by these pathways [78, 79]. In addition, both wild-type LRRK2 up-regulation and its mutations inhibit formation of the CMA translocation complex, thereby suppressing CMA activity [80, 81]. Subsequently, the normal turnover of CMA substrates is disrupted. Although LRRK2 mutants damage CMA process, the detailed relationship with neuronal loss, especially in animal models, still needs to be further explored.

In physiological conditions, about $10 \%$ of LRRK2 can present in the OMM, giving rise to the hypothesis that LRRK2 mutations might have influence over mitochondrial function. Study showed LRRK2 G2019S could lead to mitochondrial fragments by enhancing mitochondrial fission via phosphorylating decaprenyl diphosphate synthase subunit 2 Dlp1(Dlp1) [82]. Also, LRRK2 mutants activate dendritic mitochondrial clearance by autophagy in neurons [83].

\section{DJ-1}

DJ-1 belongs to the peptidase C56 family and acts as a redox-responsive cytoprotective protein. PD-related DJ-1 mutants are rare and contribute to $1-2 \%$ of autosomal recessive PD [84]. 
DJ-1 can serve as a regulator of autophagy. The effect of DJ-1 up-regulation on macroautophagy depends on cell type. In dopaminergic neurons, DJ-1 overexpression induces ERK-dependent mitophagy and protects against neurotoxin induced apoptosis [11]. Also, in mouse embryonic fibroblasts, loss of DJ-1 suppresses basal autophagy and disrupts mitochondrial dynamics [85]. However, in some cancer cells, DJ-1 deficiency activates autophagy via JNK signaling [86]. Further studies are needed to address the relationship between DJ-1/autophagy and DA neuronal loss in the context of PD.

As an anti-oxidative protein, DJ-1 also plays an important role in regulating mitochondrial function. In human neuroblastoma M17 cells, DJ-1 wild-type overexpression induces elongated mitochondria while DJ-1 mutants overexpression causes mitochondrial fragmentation. Interestingly, DLP1 knockdown in these mutant DJ-1 cells rescues mitochondrial morphology and function [87]. Also, in DJ-1 knockout cells, autophagy degradation was impaired and defective mitochondria accumulated [88]. Loss of DJ-1 leads to mitochondrial phenotypes including reduced membrane potential, increased fragmentation and accumulation of autophagic markers. Supplementing DJ-1-deficient cells with glutathione reverses both mitochondrial and autophagic changes suggesting that DJ-1 may act to maintain mitochondrial function during oxidative stress [89].

In our recent study [90], we found that DJ-1, harboring CMA specific motif, is a direct substrate of CMA and mutation inside the motif can disturb the degradation of DJ-1 through CMA. In addition, we showed UPS is not the primary mechanism responsible for DJ-1 degradation. More interestingly, CMA preferentially clears the oxidatively injured DJ-1 and the extensive accumulation of the oxidized DJ-1 monomer following a reduction of lysosomal and CMA activity affects the formation or alters the balance of DJ-1 dimer. Furthermore, CMA-DJ-1 pathway plays a critical role in maintaining mitochondrial morphology and function under stress and protects against PD related neurotoxins induced cytoxicity. Our findings suggest that CMA/DJ-1 pathway is vital for mitochondrial homeostasis and dysregulation of this pathway may explain the neuronal loss during PD pathogenesis.

\section{Recent findings on autophagy from PD human tissues}

Compared with age-matched AD and control brain samples, CMA markers LAMP2A and Hsc70 are significantly decreased in SN and amygdala of PD brains, hinting the role of autophagy in PD pathogenesis [91]. Furthermore, in peripheral blood mononuclear cells from PD patients, Hsc70 protein level is decreased in all PD groups, while glucocerebrosidase protein level is reduced only in the genetic PD groups [92]. This study suggests that Hsc70 and glucocerebrosidase may serve as a screening tool for PD diagnosis. In addition, lysosomal glucocerebrosidase protein level and enzyme activity are selectively reduced in the region with increased $\alpha$-synuclein inside the PD brain of early stage. The loss of lysosomal glucocerebrosidase is directly related to reduced lysosomal CMA activity, increased $\alpha$-synuclein and decreased ceramide, which suggests that compromise in lysosomal function contributes to PD pathology [93]. One study also shows that the fibroblasts from PD patients with the mutation G2019S have higher level of autophagy compared with wild type fibroblasts, which contributes to the more vulnerability to $\mathrm{MPP}^{+}$[76]. However, another study using the primary cultured fibroblasts from sporadic PD patients detects no changes in autophagy [94]. Further, one study uses iPSC-derived DA neurons from PD patients with GBA1 mutation and finds that these neurons has autophagic and lysosomal defects as well as increase in glucosylceramide and $\alpha$-synuclein levels [95]. Together, these findings provide evidence for a link between autophagic/lysosomal system and PD pathogenesis.

\section{Therapeutic implications}

Currently, there is no effective treatment to halt the progression of neuronal loss in PD. Thus, developing effective therapeutic strategy will be of great importance and will likely result from a better understanding the relationship between macroautophagy, CMA, mitochondrial homeostasis and PD pathogenesis.

First, since PD is characterized by the presence of abnormal protein aggregation, enhancing macroautophagy to remove the redundant proteins and/or dysfunctional organelles is being considered as a potential therapeutic strategy. In vivo, enhancing autophagic activity through TFEB or Beclin-1 overexpression protects nigral neurons against $\alpha$-synuclein-induced toxicity [96]. Coexpression of beclin-1 activated autophagy, reduced accumulation of $\alpha$-synulein, and ameliorated associated neuritic alterations. The above data support that beclin-1 could be a promising molecular target for PD treatment. In addition, some autophagy-inducing chemical agents have been shown to decrease $\alpha$-synuclein levels in cell and animal PD models [97, 98].

Furthermore, modulating CMA activity could provide benefits for PD treatment. In SH-SY5Y cells, generic overexpression of LAMP2A induces CMA activity and protectes DA neurons against $\alpha$-synuclein neurotoxicity [9]. Moreover, in rat SN, overexpression of LAMP2A via lentivirus can reduce total levels of $\alpha$-synuclein and ameliorate $\alpha$-synuclein induced neuronal degeneration and stratal terminal loss [9]. Also, our data demonstrated restoration of CMA activity is essential to maintain the normal function of neuronal survival factor MEF2D, thereby regulating ND6 transcription and promoting mitochondrial 
complex I activity, which protect DA neurons from MPTP induced neurotoxicity [36, 37]. Furthermore, retinoic acid derivatives can activate CMA to protect cells against proteotoxicity and oxidative stress [99].

Finally, considering the contribution of mitochondrial dysfunction to the pathogenesis of PD, restoring it might be a way to prevent or treat PD. The first attempt is to enhance mitophagy. In drosophila, overexpression of Parkin increases mitochondrial activity, reduces proteotoxicity and extends lifespan [100]. Also, another possible way is modulate mitochondrial biogenesis, which would rejuvenate mitochondrial pool. The peroxisome proliferator-activated receptor gamma, coactivator 1 alpha $(\mathrm{PGC}-1 \alpha)$ is a potential target. PGC- $1 \alpha$ positively regulates the expression of genes required for mitochondrial biogenesis and the cellular antioxidant responses. Also, expression of PGC-1 $\alpha$-regulated genes is low in $\mathrm{SN}$ neurons in early PD and overexpression of PGC- $1 \alpha$ could suppress ROS and neurodegeneration [101]. Thus upregulation of $\mathrm{PGC}-1 \alpha$ is a candidate neuroprotective strategy in PD. However, targeted up-regulation of PGC$1 \alpha$ via adeno-associated virus (AAV) in SN region of mice showed detrimental effect $[102,103]$. Thus, further studies need to clarify this discrepancy.

\section{Conclusion}

\section{Future challenges}

In our opinion, autophagy activity and mitochondrial homeostasis are of high importance in the pathogenesis of PD. Better understanding of the molecular interaction between autophagy and mitochondria function can give rise to novel therapeutic methods for PD prevention and amelioration. Since hypofunctional autophagy lead to protein/organelles accumulation while excessive autophagy activation can cause cell damage even autophagic cell death. The future challenges are to determine how to optimize autophagic activity to protect neurons during PD treatment. Also, challenges exist in identifying the common molecular pathway underlying the pathogenesis of multiple neurodegenerative diseases as well as the detailed molecular interaction between autophagy and mitochondrial dysfunction, especially in PD.

\section{Abbreviations}

ANT: Adenylate translocator; CMA: Chaperone-mediated autophagy; DA: Dopaminergic; DRP1: GTPase dynamic related protein 1; ETC.: Electron transport complex; LAMP2A: Lysosome associated membrane protein type 2A; LRRK2: Leucine-rich repeat kinase 2; MA: Macroautophagy; MFN1/ 2: Mitofusins 1/2; Miro: Mitochondrial Rho GTPases; MPP: Mitochondrial processing protease; MPTP: 1-methyl-4-phenyl-1,2,3,6-tetrahydropyridine; MPTP: Mitochondrial permeability transition pore; mtDNA: Mitochondrial DNA; ND6: NADH dehydrogenase 6; OMM: Outer membrane of mitochondria; PARL: Presenilin-associated rhomboid-like protease; PAS: Pre-autophagosomal structure; PD: Parkinson's disease; PGC-1a: peroxisome proliferator-activated receptor gamma, coactivator 1 alpha; PINK1: PTEN induced putative kinase 1; ROS: Reactive oxygen species; SN: Substantia nigra; UPS: Ubiquitin proteasome system; VDAC: Voltage dependent anion channel; VTA: Ventral segmental area

\section{Acknowledgements}

Not applicable.

\section{Funding}

The works were supported by National Natural Science Foundation of China (Grant No. 31371400) (Q.Y) and (Grant No. 31671060) (Q.Y).

Availability of data and materials

All data generated or analysed during this study are included in this published article (see Table 1).

\section{Authors' contributions}

BW made substantial contributions to design and draft the manuscript; NA and G-DG were involved in revising it critically; QY designed, revised the muscript; All the authors read and gave final approval of the manuscript to be published.

\section{Competing interests}

The authors declare that they have no competing interests.

\section{Consent for publication}

Not applicable.

Ethics approval and consent to participate

Not applicable.

\section{Author details}

${ }^{1}$ Department of Neurosurgery, Tangdu Hospital, The Fourth Military Medical University, No. 569 Xinsi Road, Baqiao District, Xi'an 710038, Shaanxi Province, China. ${ }^{2}$ Department of Neurology, Beth Isreal Deaconess Medical Center, Harvard Medical School, 330 Brookline Ave, Boston 02215, MA, USA.

Received: 31 August 2016 Accepted: 17 October 2016

Published online: 31 October 2016

\section{References}

1. Klein C, Westenberger A. Genetics of Parkinson's disease. Cold Spring Harb Perspect Med. 2012;2:a008888.

2. Recasens A, Dehay B, Bove J, Carballo-Carbajal I, Dovero S, Perez-Villalba A, Fernagut PO, Blesa J, Parent A, Perier $C$, et al. Lewy body extracts from Parkinson disease brains trigger alpha-synuclein pathology and neurodegeneration in mice and monkeys. Ann Neurol. 2014;75:351-62.

3. Puschmann A. Monogenic Parkinson's disease and parkinsonism: clinical phenotypes and frequencies of known mutations. Parkinsonism Relat Disord. 2013;19:407-15.

4. Kilarski LL, Pearson JP, Newsway V, Majounie E, Knipe MD, Misbahuddin A, Chinnery PF, Burn DJ, Clarke CE, Marion MH, et al. Systematic review and UK-based study of PARK2 (parkin), PINK1, PARK7 (DJ-1) and LRRK2 in early-onset Parkinson's disease. Mov Disord. 2012;27:1522-9.

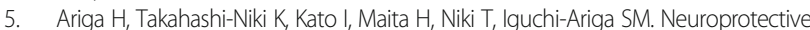
function of DJ-1 in Parkinson's disease. Oxid Med Cell Longev. 2013;2013:683920.

6. Zavodszky E, Seaman MN, Moreau K, Jimenez-Sanchez M, Breusegem SY, Harbour ME, Rubinsztein DC. Mutation in VPS35 associated with Parkinson's disease impairs WASH complex association and inhibits autophagy. Nat Commun. 2014:5:3828.

7. Nixon RA. The role of autophagy in neurodegenerative disease. Nat Med. 2013;19:983-97.

8. Oueslati A, Schneider BL, Aebischer P, Lashuel HA. Polo-like kinase 2 regulates selective autophagic alpha-synuclein clearance and suppresses its toxicity in vivo. Proc Natl Acad Sci U S A. 2013;110:E3945-54.

9. Xilouri M, Brekk OR, Landeck N, Pitychoutis PM, Papasilekas T, PapadopoulouDaifoti Z, Kirik D, Stefanis L. Boosting chaperone-mediated autophagy in vivo mitigates alpha-synuclein-induced neurodegeneration. Brain. 2013;136:2130-46.

10. Wong AS, Lee RH, Cheung AY, Yeung PK, Chung SK, Cheung ZH, Ip NY. Cdk5-mediated phosphorylation of endophilin B1 is required for induced autophagy in models of Parkinson's disease. Nat Cell Biol. 2011;13:568-79.

11. Gao H, Yang W, Qi Z, Lu L, Duan C, Zhao C, Yang H. DJ-1 protects dopaminergic neurons against rotenone-induced apoptosis by enhancing ERK-dependent mitophagy. J Mol Biol. 2012;423:232-48.

12. Osellame LD, Blacker TS, Duchen MR. Cellular and molecular mechanisms of mitochondrial function. Best Pract Res Clin Endocrinol Metab. 2012;26:711-23. 
13. Lin MT, Cantuti-Castelvetri I, Zheng K, Jackson KE, Tan YB, Arzberger T, Lees AJ, Betensky RA, Beal MF, Simon DK. Somatic mitochondrial DNA mutations in early Parkinson and incidental Lewy body disease. Ann Neurol. 2012;71:850-4.

14. Cali T, Ottolini D, Negro A, Brini M. alpha-Synuclein controls mitochondrial calcium homeostasis by enhancing endoplasmic reticulum-mitochondria interactions. J Biol Chem. 2012;287:17914-29.

15. Lee DH, Kim CS, Lee YJ. Astaxanthin protects against MPTP/MPP + -induced mitochondrial dysfunction and ROS production in vivo and in vitro. Food Chem Toxicol. 2011:49:271-80.

16. Karuppagounder SS, Madathil SK, Pandey M, Haobam R, Rajamma U, Mohanakumar KP. Quercetin up-regulates mitochondrial complex-I activity to protect against programmed cell death in rotenone model of Parkinson's disease in rats. Neuroscience. 2013:236:136-48.

17. Klionsky DJ, Abdelmohsen K, Abe A, Abedin MJ, Abeliovich H, Acevedo Arozena A, Adachi H, Adams CM, Adams PD, Adeli K, et al. Guidelines for the use and interpretation of assays for monitoring autophagy (3rd edition). Autophagy. 2016;12:1-222

18. Rabinowitz JD, White E. Autophagy and metabolism. Science. 2010;330:1344-8.

19. Pyo JO, Nah J, Jung YK. Molecules and their functions in autophagy. Exp Mol Med. 2012;44:73-80.

20. Wong AS, Cheung ZH, Ip NY. Molecular machinery of macroautophagy and its deregulation in diseases. Biochim Biophys Acta. 2011;1812:1490-7.

21. Vives-Bauza C, Przedborski S. Mitophagy: the latest problem for Parkinson's disease. Trends Mol Med. 2011;17:158-65.

22. Mijaljica $D$, Prescott $M$, Devenish RJ. Microautophagy in mammalian cells: revisiting a 40-year-old conundrum. Autophagy. 2011;7:673-82.

23. Li WW, Li J, Bao JK. Microautophagy: lesser-known self-eating. Cell Mol Life Sci. 2012:69:1125-36.

24. Petrovski G, Das DK. Does autophagy take a front seat in lifespan extension? J Cell Mol Med. 2010;14:2543-51.

25. Kaushik S, Cuervo AM. Chaperone-mediated autophagy: a unique way to enter the lysosome world. Trends Cell Biol. 2012;22:407-17.

26. Cuervo AM. Chaperone-mediated autophagy: selectivity pays off. Trends Endocrinol Metab. 2010;21:142-50.

27. Ali AB, Nin DS, Tam J, Khan M. Role of chaperone mediated autophagy (CMA) in the degradation of misfolded $\mathrm{N}$-CoR protein in non-small cell lung cancer (NSCLC) cells. PLoS One. 2011;6:e25268.

28. Cai Z, Zeng W, Tao K, E Z, Wang B, Yang Q. Chaperone-mediated autophagy: roles in neuroprotection. Neurosci Bull. 2015;31:452-8.

29. Exner N, Lutz AK, Haass C, Winklhofer KF. Mitochondrial dysfunction in Parkinson's disease: molecular mechanisms and pathophysiological consequences. EMBO J. 2012;31:3038-62.

30. Martinez TN, Greenamyre JT. Toxin models of mitochondrial dysfunction in Parkinson's disease. Antioxid Redox Signal. 2012;16:920-34.

31. Sterky FH, Hoffman AF, Milenkovic D, Bao B, Paganelli A, Edgar D, Wibom R, Lupica CR, Olson L, Larsson NG. Altered dopamine metabolism and increased vulnerability to MPTP in mice with partial deficiency of mitochondrial complex I in dopamine neurons. Hum Mol Genet. 2012;21:1078-89.

32. Ramonet D, Perier C, Recasens A, Dehay B, Bove J, Costa V, Scorrano L, Vila M. Optic atrophy 1 mediates mitochondria remodeling and dopaminergic neurodegeneration linked to complex I deficiency. Cell Death Differ. 2013;20:77-85.

33. Ivatt RM, Sanchez-Martinez A, Godena VK, Brown S, Ziviani E, Whitworth AJ. Genome-wide RNAi screen identifies the Parkinson disease GWAS risk locus SREBF1 as a regulator of mitophagy. Proc Natl Acad Sci U S A. 2014;111:8494-9.

34. Do CB, Tung JY, Dorfman E, Kiefer AK, Drabant EM, Francke U, Mountain JL, Goldman SM, Tanner CM, Langston JW, et al. Web-based genome-wide association study identifies two novel loci and a substantial genetic component for Parkinson's disease. PLoS Genet. 2011;7:e1002141.

35. Burchell VS, Nelson DE, Sanchez-Martinez A, Delgado-Camprubi M, Ivatt RM, Pogson JH, Randle SJ, Wray S, Lewis PA, Houlden H, et al. The Parkinson's disease-linked proteins $\mathrm{Fbxo7}$ and Parkin interact to mediate mitophagy. Nat Neurosci. 2013;16:1257-65.

36. She H, Yang Q, Shepherd K, Smith Y, Miller G, Testa C, Mao Z. Direct regulation of complex I by mitochondrial MEF $2 D$ is disrupted in a mouse model of Parkinson disease and in human patients. J Clin Invest. 2011;121:930-40.

37. Wang B, Cai Z, Lu F, Li C, Zhu X, Su L, Gao G, Yang Q. Destabilization of survival factor MEF2D mRNA by neurotoxin in models of Parkinson's disease. J Neurochem. 2014;130:720-8.

38. Volpicelli-Daley LA, Luk KC, Patel TP, Tanik SA, Riddle DM, Stieber A, Meaney DF, Trojanowski JQ, Lee VM. Exogenous alpha-synuclein fibrils induce Lewy body pathology leading to synaptic dysfunction and neuron death. Neuron. 2011;72:57-71.

39. Horvath I, Weise CF, Andersson EK, Chorell E, Sellstedt M, Bengtsson C, Olofsson A, Hultgren SJ, Chapman M, Wolf-Watz M, et al. Mechanisms of protein oligomerization: inhibitor of functional amyloids templates alphasynuclein fibrillation. J Am Chem Soc. 2012;134:3439-44.

40. Hansen C, Angot E, Bergstrom AL, Steiner JA, Pieri L, Paul G, Outeiro TF, Melki R, Kallunki P, Fog K, et al. alpha-Synuclein propagates from mouse brain to grafted dopaminergic neurons and seeds aggregation in cultured human cells. J Clin Invest. 2011:121:715-25.

41. Rott R, Szargel R, Shani V, Bisharat S, Engelender S. alpha-Synuclein ubiquitination and novel therapeutic targets for Parkinson's disease. CNS Neurol Disord Drug Targets. 2014;13:630-7.

42. Popova B, Kleinknecht A, Braus GH. Posttranslational Modifications and Clearing of alpha-Synuclein Aggregates in Yeast. Biomolecules. 2015;5:617-34.

43. Ebrahimi-Fakhari D, Cantuti-Castelvetri I, Fan Z, Rockenstein E, Masliah E, Hyman BT, McLean PJ, Unni VK. Distinct roles in vivo for the ubiquitinproteasome system and the autophagy-lysosomal pathway in the degradation of alpha-synuclein. J Neurosci. 2011;31:14508-20.

44. Vogiatzi T, Xilouri M, Vekrellis $K$, Stefanis L. Wild type alpha-synuclein is degraded by chaperone-mediated autophagy and macroautophagy in neuronal cells. J Biol Chem. 2008;283:23542-56.

45. Choubey V, Safiulina D, Vaarmann A, Cagalinec M, Wareski P, Kuum M, Zharkovsky A, Kaasik A. Mutant A53T alpha-synuclein induces neuronal death by increasing mitochondrial autophagy. J Biol Chem. 2011;286:10814-24.

46. Winslow AR, Rubinsztein DC. The Parkinson disease protein alpha-synuclein inhibits autophagy. Autophagy. 2011;7:429-31.

47. Tanik SA, Schultheiss CE, Volpicelli-Daley LA, Brunden KR, Lee VM. Lewy bodylike alpha-synuclein aggregates resist degradation and impair macroautophagy. J Biol Chem. 2013;288:15194-210.

48. Imaizumi Y, Okada Y, Akamatsu W, Koike M, Kuzumaki N, Hayakawa $\mathrm{H}$, Nihira T, Kobayashi T, Ohyama M, Sato S, et al. Mitochondrial dysfunction associated with increased oxidative stress and alpha-synuclein accumulation in PARK2 iPSC-derived neurons and postmortem brain tissue. Mol Brain. 2012;5:35.

49. Nakamura K, Nemani VM, Azarbal F, Skibinski G, Levy JM, Egami K, Munishkina L, Zhang J, Gardner B, Wakabayashi J, et al. Direct membrane association drives mitochondrial fission by the Parkinson disease-associated protein alpha-synuclein. J Biol Chem. 2011;286:20710-26.

50. Zhu Y, Duan C, Lu L, Gao H, Zhao C, Yu S, Ueda K, Chan P, Yang H. alphaSynuclein overexpression impairs mitochondrial function by associating with adenylate translocator. Int J Biochem Cell Biol. 2011;43:732-41.

51. Lu L, Zhang C, Cai Q, Lu Q, Duan C, Zhu Y, Yang H. Voltage-dependent anion channel involved in the alpha-synuclein-induced dopaminergic neuron toxicity in rats. Acta Biochim Biophys Sin Shanghai. 2013;45:170-8.

52. Auluck PK, Caraveo G, Lindquist S. alpha-Synuclein: membrane interactions and toxicity in Parkinson's disease. Annu Rev Cell Dev Biol. 2010;26:211-33.

53. Pemberton S, Madiona K, Pieri L, Kabani M, Bousset L, Melki R. Hsc70 protein interaction with soluble and fibrillar alpha-synuclein. J Biol Chem. 2011;286:34690-9.

54. Redeker V, Pemberton S, Bienvenut W, Bousset L, Melki R. Identification of protein interfaces between alpha-synuclein, the principal component of Lewy bodies in Parkinson disease, and the molecular chaperones human Hsc70 and the yeast Ssa1p. J Biol Chem. 2012;287:32630-9.

55. Danzer KM, Ruf WP, Putcha P, Joyner D, Hashimoto T, Glabe C, Hyman BT, McLean PJ. Heat-shock protein 70 modulates toxic extracellular alpha-synuclein oligomers and rescues trans-synaptic toxicity. FASEB J. 2011;25:326-36.

56. Yang Q, She H, Gearing M, Colla E, Lee M, Shacka JJ, Mao Z. Regulation of neuronal survival factor MEF2D by chaperone-mediated autophagy. Science. 2009;323:124-7.

57. Singleton $A B$, Farrer MJ, Bonifati $\mathrm{V}$. The genetics of Parkinson's disease: progress and therapeutic implications. Mov Disord. 2013;28:14-23.

58. Rakovic A, Grunewald A, Kottwitz J, Bruggemann N, Pramstaller PP, Lohmann K, Klein C. Mutations in PINK1 and Parkin impair ubiquitination of Mitofusins in human fibroblasts. PLoS One. 2011;6:e16746.

59. Wang H, Song P, Du L, Tian W, Yue W, Liu M, Li D, Wang B, Zhu Y, Cao C, et al. Parkin ubiquitinates Drp1 for proteasome-dependent degradation: implication of dysregulated mitochondrial dynamics in Parkinson disease. J Biol Chem. 2011;286:11649-58.

60. Vincow ES, Merrihew G, Thomas RE, Shulman NJ, Beyer RP, MacCoss MJ, Pallanck LJ. The PINK1-Parkin pathway promotes both mitophagy and 
selective respiratory chain turnover in vivo. Proc Natl Acad Sci U S A. 2013;110:6400-5.

61. Morais VA, Haddad D, Craessaerts K, De Bock PJ, Swerts J, Vilain S, Aerts L, Overbergh L, Grunewald A, Seibler P, et al. PINK1 loss-of-function mutations affect mitochondrial complex I activity via NdufA10 ubiquinone uncoupling. Science. 2014;344:203-7.

62. Bouman L, Schlierf A, Lutz AK, Shan J, Deinlein A, Kast J, Galehdar Z, Palmisano $V$, Patenge $N$, Berg $D$, et al. Parkin is transcriptionally regulated by ATF4: evidence for an interconnection between mitochondrial stress and ER stress. Cell Death Differ. 2011:18:769-82.

63. Van Laar VS, Roy N, Liu A, Rajprohat S, Arnold B, Dukes AA, Holbein CD, Berman SB. Glutamate excitotoxicity in neurons triggers mitochondrial and endoplasmic reticulum accumulation of Parkin, and in the presence of $\mathrm{N}$ acetyl cysteine, mitophagy. Neurobiol Dis. 2015;74:180-93.

64. Cali T, Ottolini D, Negro A, Brini M. Enhanced parkin levels favor ERmitochondria crosstalk and guarantee $\mathrm{Ca}(2+)$ transfer to sustain cell bioenergetics. Biochim Biophys Acta. 2013;1832:495-508.

65. Pickrell AM, Youle RJ. The roles of PINK1, parkin, and mitochondrial fidelity in Parkinson's disease. Neuron. 2015;85:257-73.

66. Scarffe LA, Stevens DA, Dawson VL, Dawson TM. Parkin and PINK1: much more than mitophagy. Trends Neurosci. 2014;37:315-24.

67. Haskin J, Szargel R, Shani V, Mekies LN, Rott R, Lim GG, Lim KL, Bandopadhyay $\mathrm{R}$, Wolosker $H$, Engelender S. AF-6 is a positive modulator of the PINK1/parkin pathway and is deficient in Parkinson's disease. Hum Mol Genet. 2013;22:2083-96.

68. Lazarou M, Jin SM, Kane LA, Youle RJ. Role of PINK1 binding to the TOM complex and alternate intracellular membranes in recruitment and activation of the E3 ligase Parkin. Dev Cell. 2012;22:320-33.

69. Lubbe S, Morris HR. Recent advances in Parkinson's disease genetics. J Neurol. 2014;261:259-66

70. Dusonchet J, Kochubey O, Stafa K, Young Jr SM, Zufferey R, Moore DJ, Schneider BL, Aebischer P. A rat model of progressive nigral neurodegeneration induced by the Parkinson's disease-associated G2019S mutation in LRRK2. J Neurosci. 2011:31:907-12.

71. Tong Y, Giaime E, Yamaguchi H, Ichimura T, Liu Y, Si H, Cai H, Bonventre JV, Shen J. Loss of leucine-rich repeat kinase 2 causes age-dependent bi-phasic alterations of the autophagy pathway. Mol Neurodegener. 2012;7:2.

72. Manzoni C, Mamais A, Dihanich S, Abeti R, Soutar MP, Plun-Favreau H, Giunti P, Tooze SA, Bandopadhyay R, Lewis PA. Inhibition of LRRK2 kinase activity stimulates macroautophagy. Biochim Biophys Acta. 2013;1833:2900-10

73. Gomez-Suaga P, Luzon-Toro B, Churamani D, Zhang L, Bloor-Young D, Patel S, Woodman PG, Churchill GC, Hilfiker S. Leucine-rich repeat kinase 2 regulates autophagy through a calcium-dependent pathway involving NAADP. Hum Mol Genet. 2012;21:511-25.

74. Ramonet D, Daher JP, Lin BM, Stafa K, Kim J, Banerjee R, Westerlund M, Pletnikova O, Glauser L, Yang L, et al. Dopaminergic neuronal loss, reduced neurite complexity and autophagic abnormalities in transgenic mice expressing G2019S mutant LRRK2. PLoS One. 2011;6:e18568.

75. Winner B, Melrose HL, Zhao C, Hinkle KM, Yue M, Kent C, Braithwaite AT, Ogholikhan S, Aigner R, Winkler J, et al. Adult neurogenesis and neurite outgrowth are impaired in LRRK2 G2019S mice. Neurobiol Dis. 2011:41:706-16

76. Yakhine-Diop SM, Bravo-San Pedro JM, Gomez-Sanchez R, Pizarro-Estrella E, Rodriguez-Arribas M, Climent V, Aiastui A, Lopez de Munain A, Fuentes JM, Gonzalez-Polo RA. G2019S LRRK2 mutant fibroblasts from Parkinson's disease patients show increased sensitivity to neurotoxin 1-methyl-4phenylpyridinium dependent of autophagy. Toxicology. 2014;324:1-9.

77. Bravo-San Pedro JM, Niso-Santano M, Gomez-Sanchez R, Pizarro-Estrella E, Aiastui-Pujana A, Gorostidi A, Climent V, Lopez de Maturana R, SanchezPernaute R, Lopez de Munain A, et al. The LRRK2 G2019S mutant exacerbates basal autophagy through activation of the MEK/ERK pathway. Cell Mol Life Sci. 2013;70:121-36.

78. Orenstein SJ, Kuo SH, Tasset I, Arias E, Koga H, Fernandez-Carasa I, Cortes E, Honig LS, Dauer W, Consiglio A, et al. Interplay of LRRK2 with chaperonemediated autophagy. Nat Neurosci. 2013;16:394-406.

79. Lichtenberg M, Mansilla A, Zecchini VR, Fleming A, Rubinsztein DC. The Parkinson's disease protein LRRK2 impairs proteasome substrate clearance without affecting proteasome catalytic activity. Cell Death Dis. 2011;2:e196.

80. Yue Z, Yang XW. Dangerous duet: LRRK2 and alpha-synuclein jam at CMA. Nat Neurosci. 2013;16:375-7.
81. Sweet ES, Saunier-Rebori B, Yue Z, Blitzer RD. The Parkinson's DiseaseAssociated Mutation LRRK2-G2019S Impairs Synaptic Plasticity in Mouse Hippocampus. J Neurosci. 2015;35:11190-5.

82. Niu J, Yu M, Wang C, Xu Z. Leucine-rich repeat kinase 2 disturbs mitochondrial dynamics via Dynamin-like protein. J Neurochem. 2012;122:650-8.

83. Cherra 3rd SJ, Steer E, Gusdon AM, Kiselyov K, Chu CT. Mutant LRRK2 elicits calcium imbalance and depletion of dendritic mitochondria in neurons. Am J Pathol. 2013;182:474-84

84. Rochet JC, Hay BA, Guo M. Molecular insights into Parkinson's disease. Prog Mol Biol Transl Sci. 2012;107:125-88.

85. Krebiehl G, Ruckerbauer S, Burbulla LF, Kieper N, Maurer B, Waak J, Wolburg H, Gizatullina Z, Gellerich FN, Woitalla D, et al. Reduced basal autophagy and impaired mitochondrial dynamics due to loss of Parkinson's diseaseassociated protein DJ-1. PLoS One. 2010;5:e9367.

86. Ren H, Fu K, Mu C, Li B, Wang D, Wang G. DJ-1, a cancer and Parkinson's disease associated protein, regulates autophagy through JNK pathway in cancer cells. Cancer Lett. 2010;297:101-8.

87. Wang X, Petrie TG, Liu Y, Liu J, Fujioka H, Zhu X. Parkinson's diseaseassociated DJ-1 mutations impair mitochondrial dynamics and cause mitochondrial dysfunction. J Neurochem. 2012;121:830-9.

88. Thomas KJ, McCoy MK, Blackinton J, Beilina A, van der Brug M, Sandebring A, Miller D, Maric D, Cedazo-Minguez A, Cookson MR. DJ-1 acts in parallel to the PINK1/parkin pathway to control mitochondrial function and autophagy. Hum Mol Genet. 2011;20:40-50.

89. McCoy MK, Cookson MR. DJ-1 regulation of mitochondrial function and autophagy through oxidative stress. Autophagy. 2011;7:531-2.

90. Wang B, Cai Z, Tao K, Zeng W, Lu F, Yang R, Feng D, Gao G, Yang Q. Essential control of mitochondrial morphology and function by chaperone-mediated autophagy through degradation of PARK7. Autophagy. 2016;12:1215-28.

91. Alvarez-Erviti L, Rodriguez-Oroz MC, Cooper JM, Caballero C, Ferrer I, Obeso JA, Schapira AH. Chaperone-mediated autophagy markers in Parkinson disease brains. Arch Neurol. 2010;67:1464-72.

92. Papagiannakis N, Xilouri M, Koros C, Stamelou M, Antonelou R, Maniati M, Papadimitriou D, Moraitou M, Michelakakis H, Stefanis L. Lysosomal alterations in peripheral blood mononuclear cells of Parkinson's disease patients. Mov Disord. 2015:30:1830-4.

93. Murphy KE, Gysbers AM, Abbott SK, Tayebi N, Kim WS, Sidransky E, Cooper A, Garner B, Halliday GM. Reduced glucocerebrosidase is associated with increased alpha-synuclein in sporadic Parkinson's disease. Brain. 2014;137:834-48.

94. Ambrosi G, Ghezzi C, Sepe S, Milanese C, Payan-Gomez C, Bombardieri CR, Armentero MT, Zangaglia R, Pacchetti $C$, Mastroberardino PG, et al. Bioenergetic and proteolytic defects in fibroblasts from patients with sporadic Parkinson's disease. Biochim Biophys Acta. 2014;1842:1385-94.

95. Schondorf DC, Aureli M, MCAllister FE, Hindley CJ, Mayer F, Schmid B, Sardi SP, Valsecchi M, Hoffmann S, Schwarz LK, et al. iPSC-derived neurons from GBA1-associated Parkinson's disease patients show autophagic defects and impaired calcium homeostasis. Nat Commun. 2014:5:4028.

96. Decressac M, Mattsson B, Weikop P, Lundblad M, Jakobsson J, Bjorklund A. TFEB-mediated autophagy rescues midbrain dopamine neurons from alphasynuclein toxicity. Proc Natl Acad Sci U S A. 2013;110:E1817-26.

97. Shoji-Kawata S, Sumpter R, Leveno M, Campbell GR, Zou Z, Kinch L, Wilkins AD, Sun Q, Pallauf K, MacDuff D, et al. Identification of a candidate therapeutic autophagy-inducing peptide. Nature. 2013;494:201-6.

98. Steele JW, Ju S, Lachenmayer ML, Liken J, Stock A, Kim SH, Delgado LM, Alfaro IE, Bernales S, Verdile G, et al. Latrepirdine stimulates autophagy and reduces accumulation of alpha-synuclein in cells and in mouse brain. Mol Psychiatry. 2013;18:882-8.

99. Anguiano J, Garner TP, Mahalingam M, Das BC, Gavathiotis E, Cuervo AM. Chemical modulation of chaperone-mediated autophagy by retinoic acid derivatives. Nat Chem Biol. 2013:9:374-82.

100. Rana A, Rera M, Walker DW. Parkin overexpression during aging reduces proteotoxicity, alters mitochondrial dynamics, and extends lifespan. Proc Natl Acad Sci U S A. 2013;110:8638-43.

101. St-Pierre J, Drori S, Uldry M, Silvaggi JM, Rhee J, Jager S, Handschin C, Zheng $K$, Lin J, Yang $W$, et al. Suppression of reactive oxygen species and neurodegeneration by the PGC-1 transcriptional coactivators. Cell. 2006;127:397-408. 
102. Clark J, Silvaggi JM, Kiselak T, Zheng K, Clore EL, Dai Y, Bass CE, Simon DK. Pgc-1alpha overexpression downregulates Pitx3 and increases susceptibility to MPTP toxicity associated with decreased Bdnf. PLoS One. 2012;7:e48925.

103. Ciron C, Lengacher S, Dusonchet J, Aebischer P, Schneider BL. Sustained expression of PGC-1alpha in the rat nigrostriatal system selectively impairs dopaminergic function. Hum Mol Genet. 2012;21:1861-76.

Submit your next manuscript to BioMed Central and we will help you at every step:

- We accept pre-submission inquiries

- Our selector tool helps you to find the most relevant journal

- We provide round the clock customer support

- Convenient online submission

- Thorough peer review

- Inclusion in PubMed and all major indexing services

- Maximum visibility for your research

Submit your manuscript at www.biomedcentral.com/submit 